\title{
Quantum Space and the Evolution of the Dark Universe
}

\author{
Carlos A. Melendres \\ The SHD Institute \\ 216 F Street, Davis, CA 95616, U.S.A. \\ Email: camelendres@SHDInstitute.org
}

August 2017

Keywords: quantum space, evolution of the universe, dark energy, thermodynamics, dark matter, plasma, recombination, vacuum energy, cosmological constant, Quintessence, spaceons 


\title{
Quantum Space and the Evolution of the Dark Universe
}

\author{
Carlos A. Melendres
}

\begin{abstract}
We present a model of space that considers it to be a quantized dynamical entity which is a component of the universe along with matter and radiation. The theory is used together with thermodynamic data to provide a new view of cosmic evolution and an insight into the nature of dark energy and dark matter.

Space is made up of energy quanta. The universe started from an atomic size volume at very high temperature and pressure near the Planck epoch. Upon expansion and cooling, phase transitions occurred resulting in the formation of radiation, fundamental particles, and matter. These nucleate and grow into stars, galaxies, and clusters. From a phase diagram of cosmic composition, we obtained a correlation between dark energy and the energy of space. Using the Friedmann equations, data from WMAP studies of the composition of the universe at $3.0 \times 10^{5}\left(\mathrm{a}=5.25 \times 10^{-2}\right)$ years and at present $(\mathrm{a}=1)$, are well fitted by our model with an equation of state parameter, $\mathrm{w}=-0.7$. The accelerated expansion of the universe, starting at about 7 billion years, determined by BOSS measurements, also correlates well with the dominance of dark energy at $7.25 \times 10^{9}$ years $(a=0.65)$. The expansion can be attributed to Quintessence with a space force arising from a quantum space field. From our phase diagram, we also find a correlation suggesting that dark matter is a plasma form of matter similar to that which existed during the photon epoch immediately prior to recombination.

Our Quantum Space Model leads to a universe which is homogeneous and isotropic without the need for inflation. The thermodynamics of expansion is consistent with BOSS data that show the process to be adiabatic and the rate of expansion decelerating during the first 6 billion years after the Big Bang. However, it became non-adiabatic and accelerating thereafter. This implies an influx of energy from a source outside the universe; it warrants consideration as a possible factor in the accelerated expansion of the universe.
\end{abstract}




\section{INTRODUCTION}

The nature of space has been the subject of debate among philosophers, scientists, and mathematicians from Plato to Descartes, Kant, Newton, Leibniz, and others [1]. Space is generally viewed like a canvas where nature's landscape and events are portrayed; it is then treated geometrically and mathematically as a surface in 4-dimensional space-time. Still we understand little of space. Here we propose a descriptive physical model of space that considers it as a quantized dynamical entity which is an active participant in the evolution of the universe and one of its major components, along with matter and radiation. The model provides a mechanism to explain the accelerated expansion of the universe due to dark energy, as well as the nature of dark matter. It also leads to a more natural model of the evolution of the universe.

\section{A MODEL OF SPACE AND COSMIC EVOLUTION}

It has been thought of that space is not truly empty, that vacuum contains virtual particles that pop in and out of existence, and used to explain the Casimir effect [2]. It seems reasonable to take a view that space contains energy and is quantized. In our model, we consider space to consist of quanta that propagate as waves and can be described by the Planck quantum energy expression:

$$
\mathrm{E}=\mathrm{hc} / \lambda
$$

The symbols have their usual meaning: $\mathrm{E}$, is the energy; $\lambda$, the wavelength, and $\mathrm{h}$, the Planck constant. The expression defines the equivalence of space and energy, in analogy to the relation between energy and mass, given by Einstein's equation, $\mathrm{E}=\mathrm{mc}^{2}$. Thus, energy is inherent in space and space in energy. We shall call the quantal units of space as "spaceons". The space waves (spaceons) can be thought of as the carrier of energy and contain the universe. From the principle of wave- particle duality, the spaceons can also be thought of as a gas which obeys the equation of state [3],

$$
\mathrm{PV}=\mathrm{N}_{0} \mathrm{~K}_{\beta} \mathrm{T} \text {. }
$$

$\mathrm{P}$ is the pressure of the gas, $\mathrm{V}$ the volume, $\mathrm{T}$ the temperature, $\mathrm{N}$ the Avogadro and $\mathrm{K}_{\beta}$ the Boltzman constant.

With this theory we can model the gross features of the evolution of the universe as follows: The universe started (at time, $\mathrm{t}=0$ ) as a very small volume of compressed gas (the spaceons) at an extremely high pressure and temperature. For example, an Avogadro number of gas particles occupying a volume of $4.2 \times 10^{-33} \mathrm{~m}^{3}$ (a sphere of $0.1 \mathrm{~A}^{0}$ radius), at a pressure of $1.976 \times 10^{65} \mathrm{~Pa}$ will be at conditions during the Planck epoch with a temperature of $10^{32} \mathrm{~K}$. We shall refer to this initial state where the universe consisted of hot spaceons only, as the quantum space epoch. The spaceons then expand and cool as they propagate and create space. Unlike the usual expansion of a gas inside a container, the spaceons expand into "nothingness" as they propagate and create space. Note that the process is not the normal expansion of a gas against a surrounding ambient pressure, $\mathrm{P}$; it is more akin to effusion into a vacuum.

In the process, the spaceons cool to appropriate threshold temperatures where phase transitions occur resulting in the formation of radiation, fundamental particles, nuclei, and atoms. Gravitation causes the formation of galaxies and stars; their clumping results in clusters, local groups and superclusters. Details of the events and transformations involved at the various epochs of the evolution of the universe are similar to that of the Big Bang model and are well documented in books on Cosmology $[4,5,6]$. They need not be repeated here. The universe with radiation, matter, and spaceons continues to expand as we know it. 
Our model is simpler than that of the Big Bang. It is more like a " hiss" rather than a "bang" (which is thought of as a violent explosion with debris of matter flying out all over). Evolution of the universe started at an earlier epoch when quantum space dominated. A Theory of Inflation is not necessary to form a universe which is homogeneous and isotropic. Space and radiation were first created from which matter formed subsequently. The processes of expansion of spaceons and their phase transformation (nucleation and growth) to form matter occured in an orderly fashion, following well known physico-chemical processes (This is analogous to the formation of snow and ice, on a cold winter day).

\section{RESULTS AND DISCUSSION}

\subsection{Composition of the Universe}

Several methods are used to determine the composition of the universe. Results of the WMAP satellite studies [7] gave the composition shown in Table I soon after the Big Bang and at present:

Table I-Composition of the Universe

At the Big Bang

Dark Matter

Dark Energy

Neutrinos

Photons
Ordinary Matter

At Present (13.8 billion years after)
$63 \%$

$1 \overline{2} \%$

$10 \%$

$15 \%$
$24 \%$

$71.4 \%$

$4.6 \%$
The composition during the cosmic evolution is also well documented [4,5,6].Table II shows this as a function of pressure $(\mathrm{P})$ and temperature $(\mathrm{T})$ at various times and Cosmic Epochs.

Table II.-Composition of the Universe during its Evolution

Time (after Big Bang)/ Temperature (K) Pressure (Pa) Composition

(Cosmic Epoch)

$10^{-12}$ to $0.02 \mathrm{sec} /$

(Quark,Hadron,Lepton,

$$
\begin{array}{ccc}
10^{11}-10^{9} & 10^{32}-10^{27} \quad \begin{array}{c}
\text { I. Fundamental particles } \\
+ \text { radiation }
\end{array}
\end{array}
$$

Electrons,Protons,Neutrons)

0.02 to $300 \mathrm{sec} /$

$$
10^{9}-10^{8}
$$

$10^{27}-10^{15}$

(Nucleosynthesis)

$300 \mathrm{sec}$ to $3.8 \times 10^{5} \mathrm{yrs} /$

$10^{8}-10^{3}$

$10^{15}-10^{-11}$

(Photon to Recombination)

$3.8 \times 10^{5}$ to $10^{9} \mathrm{yrs} /$

$3 \times 10^{3}-4$

$10^{-11}-10^{-22}$

(Dark Ages to Matter

dominated)

\section{Nuclei of H, He,D,Li}

+ radiation

III. Plasma of ionized $\mathrm{H}$, $\mathrm{He}$ and $\mathrm{e}-$

IV.Matter in galaxies,stars space, planets-gases,plasma, solid 
$10^{9}$ to $13.8 \times 10^{9} \mathrm{yrs} /$

$10^{-22}$

V. Dark Energy

(Dark Energy dominated)

At temperatures above $10^{12} \mathrm{~K}$ and time $<10^{-12} \mathrm{sec}$ (the quantum space epoch), the universe was a primeval hot gas of spaceons. Upon effusion/expansion and cooling radiation and fundamental particles (quarks, leptons, hadrons, protons, neutrons, electrons) were created. Further cooling to about $10^{9} \mathrm{~K}$, nucleosynthesis occurred to produce nuclei of $\mathrm{H}, \mathrm{He} \mathrm{Li}$ and $\mathrm{D}$. At about $10^{8} \mathrm{~K}$ a plasma phase was formed which consisted of electrons and positive, ions of $\mathrm{H}$ and He. Radiation (photons, neutrinos) was ever present and dominated the early epoch of the universe. Further cooling of the plasma until about $3 \times 10^{3} \mathrm{~K}$ resulted in recombination of electrons with positive ions of $\mathrm{H}$ and $\mathrm{He}$, converting the plasma to gases. As the universe continued to expand and cool, matter continued to form which, through the action of gravity, became galaxies, stars, and clusters. The universe cooled to $2.7 \mathrm{~K}$ as indicated by the Cosmic Microwave background. The primary constituents of the universe are: gases $(\mathrm{H}, \mathrm{He})$, plasma of electrons, protons, and He ions, ordinary matter (gasses, solids, dust, in stars, galaxies, clusters, intergalactic space) and spaceons ( gas at all temperatures); radiation is ever present.

Thermodynamics is a very powerful method for obtaining compositional information [8]. From data on composition as a function of time (cooling curves) one can construct a phase

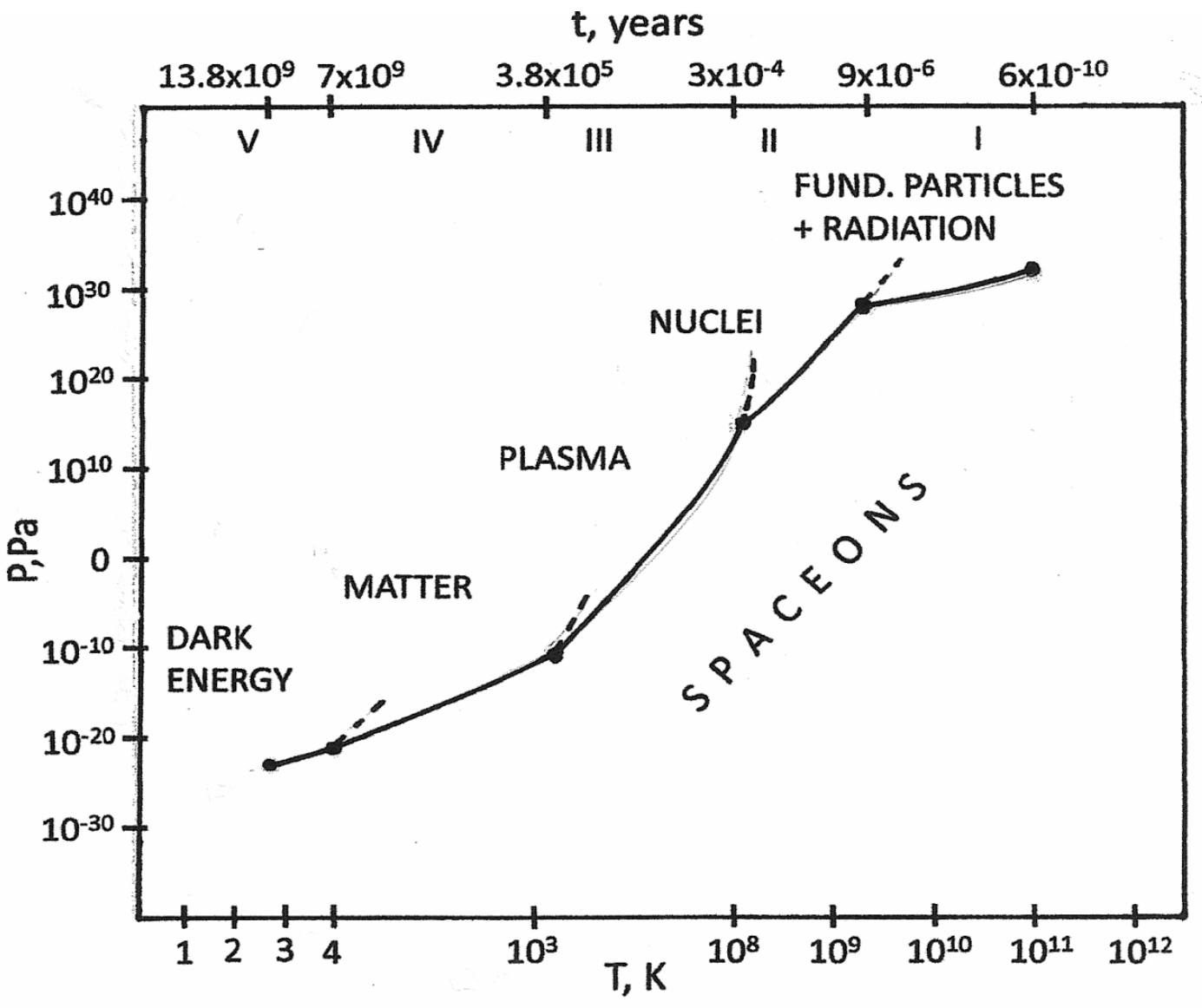

Fig. 1-Schematic Phase Diagram of the Universe with Spaceons 
diagram (similar to the well known water phase diagrams with gas, liquid, solid $\mathrm{H}_{2} \mathrm{O}$ of different crystalline forms). While such diagrams are constructed normally for the components of matter, the equivalence of matter and energy, allows one to do the same for the universe as a system. Dark energy and dark matter are components of the universe. We have constructed a thermodynamic phase diagram [8] for our model universe with spaceons using the data in Table II.

The result is shown in Fig.1. (Note that the figure is schematic and the temperature is not drawn to scale in order to highlight the phases formed). The major phases are: matter (IV, solid, gasses, plasma formed in galaxies, stars, clusters), a plasma phase (III) formed immediately after nucleosynthesis, dark energy (V) and spaceons (gas). The broken lines indicate the overlap between various phases during the process of formation. Dark matter is not indicated since its nature remains unknown. All phases are in contact with spaceons at all epochs of cosmic evolution. This is as it should be since space is in contact with all elements of the universe at all times. At extremely high temperatures and pressures (right end), fundamental particles and radiation are indistinguishable from the hot spaceons; while at low temperature/pressure (left end), dark energy overlaps with the cold spaceons. These end points are called critical points, where the 2 phases co-exist. In the case of water, for example, at the critical temperature of 647 $\mathrm{K}$ and pressure of $2.2064 \times 10^{7} \mathrm{~Pa}$, liquid water is indistinguishable from its vapor phase.

\subsection{Dark Energy and the Expansion of the Universe}

Our universe is essentially dark, consisting of only $0.5 \%$ luminous matter (in 5\% ordinary matter) and the remaining 95\% are two components, dark energy (71\%) and dark matter $(24 \%)$. Present understanding of the latter two is very poor, causing them to be dubbed "mysterious" $[9,10]$. Dark energy is hypothesized to be an unknown form of energy that permeates all of space uniformly. It is invisible and difficult to study because it does not interact with radiation, hence cannot be investigated spectroscopically. It only interacts with gravity. Its density is very low, less than ordinary or dark matter, it converts to dark matter, was less in the past than at present, and it is thought to function like an anti-gravity force. It is believed to be a property of space and causes the accelerated expansion of the universe $[9,10,11]$.

Based on our model, the expansion of the universe could be thought of as the effusion/expansion of space (spaceons) into "nothingness" in order to propagate and create more space. This appears to be a perfectly natural process driven by a pressure gradient between the "filled" space (at P) and the surrounding "nothing". It can also be thought of as driven by the pressure and temperature differential of the hot (short $\lambda_{s}$ ) and cold (long $\lambda_{s}$ ) gas states. In any case, the expansion can be viewed from the stand point of Quantum Field Theory [12], as arising from a force that is associated with a field. The latter is the space field from which emanate space quanta (analogous to the gravitational field with its associated gravitons). It is a scalar field, termed "Quintessence", and has been theorized to be the substance that comprises Dark Energy [10].

One can see from our thermodynamic phase diagram (Fig.1) that dark energy is a phase that overlaps with a new entity that we have introduced as a component of the universe, i.e., the spaceons; the two phases are indistinguishable. Hence, dark energy can be associated with spaceons, the energy of space. The amount of dark energy soon after the Big Bang was relatively small (Table I) because most of the energy of space has been converted to radiation, 
fundamental particles, dark matter and ordinary matter. Its density remains low as the universe expands.

Our model universe with spaceons is well suited for mathematical treatment using the Friedmann-Lemaitre-Robertson-Walker (FLRW) metric [11].The second Friedmann equation $[10,11]$ delineates the contributions of the various components of the universe (matter, radiation, and vacuum) to the acceleration of expansion of the universe, i.e.,

$$
\mathrm{d}^{2} \mathrm{a} / \mathrm{dt}^{2}=-(8 \pi \mathrm{Ga} / 3)\left\{(1 / 2) \rho_{\mathrm{m}}+\rho_{\mathrm{r}}-\Lambda / 8 \pi \mathrm{G}\right\}
$$

We have left out the curvature term normally appearing in 3 ), assuming that the geometry of the universe is flat $(\mathrm{k}=0$. In the above equation, $\mathrm{a}$ is the scale parameter, $\mathrm{G}$ the gravitational constant; $\rho_{\mathrm{m}}$ is the mass-energy density of matter, $\rho_{\mathrm{r}}$ that of radiation, and $\Lambda$, the cosmological constant which represents the energy of the vacuum [10]. Based on our model of space, the term "vacuum energy" is no longer a meaningful term to use; it is preferable to use the term "energy of space". The cosmological constant will then be replaced by the energy density of space, i.e.

$$
\mathrm{d}^{2} \mathrm{a} / \mathrm{dt}^{2}=-(8 / 3) \pi \mathrm{Ga}\left\{(1 / 2) \rho_{\mathrm{m}}+\rho_{\mathrm{r}}+\left(1+3 \mathrm{w}_{\mathrm{s}}\right) \rho_{\mathrm{s}}\right\}
$$

$\rho_{\mathrm{s}}$ is the energy density of space and is related to the pressure $P_{s}$ via the equation of state $P_{s}=w_{s} \rho_{s}$; $\mathrm{W}_{\mathrm{s}}$ is the equation of state parameter. A negative pressure would give rise to a positive term for the contribution of $\rho_{\mathrm{s}}$ that can cause an acceleration of the expansion. The negative pressure follows from the first law of thermodynamics for an adiabatic expansion process which is accompanied by an increase in internal energy of the system. This seems to be the situation of the universe at the present time, although the expansion may not necessarily be adiabatic (this will be discussed later) .

For the purpose of fitting the observed data (Table I), it is preferable to use another form of the Friedmnan equation. It is common to utilize one involving the Hubble constant, H, which is measured in experiments and to use velocity, da/dt, rather than acceleration $[9,10,11]$. Thus,

$$
\mathrm{a} \mathrm{H}^{2}=(\mathrm{da} / \mathrm{dt})^{2} \mathrm{a}^{2}=\left(\mathrm{H}_{0}\right)^{2}\left[\Omega_{\mathrm{m}} \mathrm{a}^{-3}+\Omega_{\mathrm{r}} \mathrm{a}^{-4}+\Omega_{\mathrm{s}} \mathrm{a}^{-3(1+\mathrm{w})}\right]
$$

where: $\mathrm{H}$ is the Hubble constant, $\mathrm{H}_{0}$ its value at the present time; $\Omega_{\mathrm{i}}$ is the density parameter for component $i$ and is given by the quantity, the fractional energy density, $\Omega_{i}=\varrho_{i} / \varrho_{c} ; \varrho_{i}$ is the energy density of $\mathrm{i}$ and $\varrho_{c}$ is the critical energy density of the universe (when $\mathrm{k}=0$ ) .

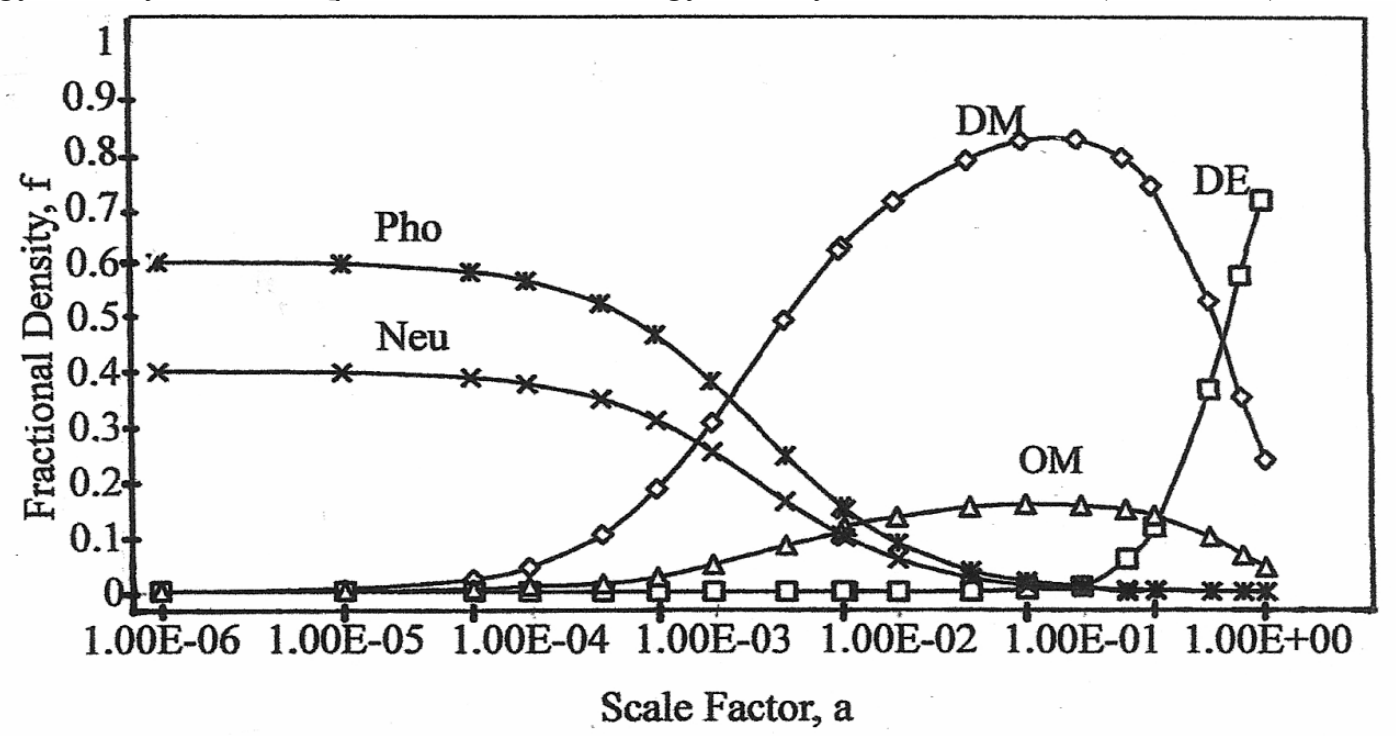

Fig.2- Fractional Energy Density (f) vs Scale Factor (a), fit with $\mathrm{ws}_{\mathrm{s}}=-0.7$ DE-Dark Energy; DM-Dark Matter; OM-Ordinary Matter; Pho-Photons; Neu-Neutrinos 
A plot of fractional energy density as a function of of "a" can be constructed to fit the measured composition of the universe at about the time of the Big Bang and at the present time (see Table 1)

The plot in Fig. 2 shows the evolution of the composition of the universe. The dominance of radiation, matter, and dark energy at various times are amply illustrated. As can be seen there is good fit of the data summarized in Table $\mathrm{I}$ at time $\mathrm{t}=13.8$ billion years $(\mathrm{a}=1)$ and at the time of the Big Bang, at about $\mathrm{t}=380,000$ years $(\mathrm{a}=5.25 \mathrm{E}-02)$, with an equation of state parameter, $\mathrm{w}_{\mathrm{s}}=-$ 0.7. Dark Energy is very small in the early universe as most of the energy is used in the creation of radiation and particles. As the universe expands, however, the energy densities of radiation and matter continue to dilute and are eventually overcome by the energy density of Dark Energy. This occurs at around 7 billion years at which time the expansion of the universe starts to accelerate. This result has been established by baryon acoustic oscillation measurements in the BOSS project [13] . Fig.3 lends support to these findings. One sees that the energy density of Dark Energy crosses that of the total energy of matter (shown by the the broken line) at $a=0.65$ $\left(\mathrm{t}=7.25 \times 10^{9}\right.$ years $)$.

Siegel [14] has obtained a similar result using a cosmological constant. However, it leads to some perflexing questions. The cosmological constant is considered to be equal to the energy of the vacuum. Calculations give a 120 orders of magnitude for the calculated value of the energy density, which is clearly catastrophically wrong. Moreover, the constancy of dark energy in the course of cosmic evolution is also thought to be unlikely [15]. Quintessence appears to be a more logical explanation for dark energy.

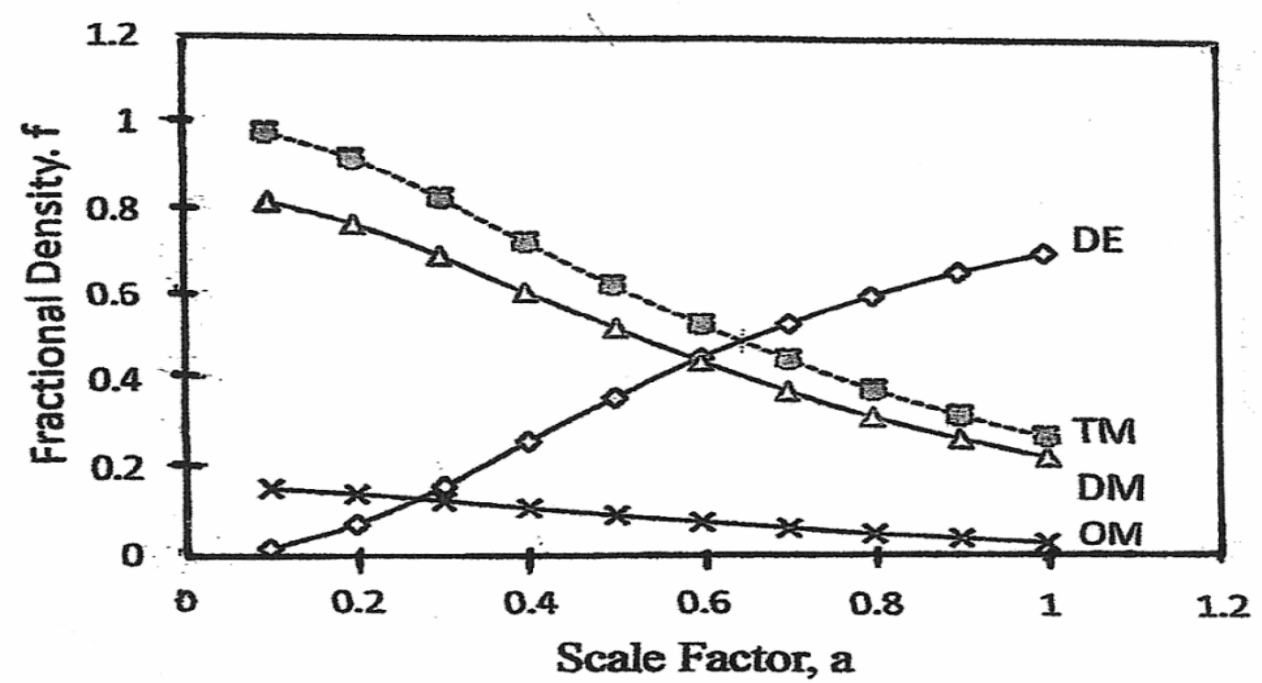

Fig.3 - Fractional Energy Density (f) vs Scale Factor (a); $\mathrm{w}_{\mathrm{s}}=-0.7$ DE- Dark Energy; DM-Dark Matter; OM-Ordinary Matter; TM- Total Matter

\subsection{THERMODYNAMICS OF EXPANSION}

In our model, the expansion of the universe is assumed to be adiabatic, i.e. with no energy transfer in and out of the system. Thus,

$$
\mathrm{Q}=\mathrm{dE}+\mathrm{PdV}=0 \text {, }
$$


where $\mathrm{Q}$ is the energy flowing into or out of the system, $\mathrm{dE}$ the change in internal energy, $\mathrm{P}$ the pressure, and $\mathrm{dV}$ the change in volume during the expansion. The work to create space, PdV, is done at the expense of the internal energy, $E$, i.e. $d E=-P d V$ If the energy stored in the Quintessence space field is finite, then the expansion would decelerate and result eventually in a "big crunch" for the universe. Our model is consistent with data from BOSS studies showing [13] the rate of expansion to be slowing down during the first 6 billion years after the Big Bang. It reached a minimum at around 7 billion years ago and then increased thereafter. $\mathrm{dE}$ and $\mathrm{dV}$ are both increasing. Thermodynamically, this means that the expansion process is no longer adiabatic, i.e. Q is not equal to zero. There is then the possibility that the accelerated expansion of the universe occurred with an "injection" or "leak"of energy from the outside. Since this is also the time when dark energy became dominant, it brings into question the relation between the two. This matter needs further investigation. In any case, one consequence of a continued supply of energy is to sustain the expansion which would eventually lead to the "cold death" of the universe.

\section{NATURE AND PROPERTIES OF SPACE QUANTA}

Our quantum model of space provides a physical picture of space. Our conception of the theory suggests some properties of space and space quanta:

1).They differ from electromagnetic radiation, hence cannot be detected by spectroscopic techniques. The electromagnetic field carries charge while the space field does not.

2). Space also differs from gravity. The space force exerts a pressure opposite to that of gravity; it is repulsive while gravity is an attractive force between material objects. The space force is sometimes referred to as an antigravitational force. However, the latter has never been been observed before. The space force appears to be distinctly different and peculiar to space.

3).The space field fits well the description of a scalar field in Quantum Field Theory. It is analogous to the gravitational field. It differs from the vector field of electromagnetic waves. It might be that the space field is the "mother" field, from which arose both force fields and matter fields [12]. Recall that at the "beginning", there were only energy and space from which were "born" radiation and matter. Thus an era of quantum space predates that of quantum gravity.

4).The space waves (spaceons) propagate space and transport energy in the universe. From this energy emanate radiation and all matter. Space also probably acts as the container and reservoir of energy in the universe. Virtual particles can pop in and out of space. When particles and antimatter annihilate, they disappear and the energy goes back to space.

\section{ON THE NATURE OF DARK MATTER}

Finally, we might say a few words about the other component of our invisible universe, dark matter, since its known characteristics match well those of a component present in our Phase Diagram (Fig.1). Dark matter [16] comprises $84 \%$ of the total mass of matter in the universe. At present, it makes up $24 \%$ of the total mass-energy of the universe It remains a mysterious, hypothetical form of matter.

Among properties that have been reported are:

1). it neither emits or absorbs electromagnetic radiation, hence it is difficult to study.

2). it moves without friction. 
3). it can only be detected through its gravitational effects on the motion of galaxies.

4). it is spread over large areas, like a cloud, and forms a "halo" around galaxies and clusters; its density decreasing as one moves away from the center [17].

5). it is also found in filaments between galaxies and clusters [18]. It has been observed that ordinary matter traces the path of dark matter; this has been attributed to a strong interaction between ordinary matter and dark matter.

We proceed with the premise that all components of universe formed during cosmic evolution and would have left their footprints in the sands of time, e.g, the CMB. The phase diagram (Fig.1) shows that the major phases in the formation of the universe are dark energy, ordinary matter (gasses, solids), and plasma. Any present-day component of the universe must originate from one of these phases. From the observed properties of dark matter, and known characteristics of plasma [19], it appears reasonable to make the assignment that dark matter corresponds to the plasma form of matter that existed during the photon epoch, just before recombination. The following facts support this contention:

1). WMAP data, mentioned earlier, show that dark matter constitutes the major component of the universe soon after the Big Bang. During this time, the universe consisted of a hot plasma of electrons, and ionized $\mathrm{H}$ and He. It was opaque and cannot emit or absorb light. This state persisted until about 380,000 years after the Big Bang at which time recombination took place and the universe became transparent.

2). Other characteristics of plasmas match exactly those of dark matter. Plasma, like dark matter, hangs around like a cosmic fog around galaxies and clusters, making it invisible and difficult to characterize.

3). That ordinary matter traces the path of dark matter can be explained by the fact that upon recombination of electrons and positive ions in the dark matter plasma, ordinary matter is formed; the plasma evaporates into $\mathrm{H}$ and He gasses. Thus ordinary matter follows the trail of dark matter.

4). Filamentation is a characteristic of plasmas; they move without friction, since the ions do not have attractive interaction and move collectively instead [19]. This lack of interaction also explains the origin of dark matter halos [17] that hover around galaxies.

5). The dark matter plasma scatters elastically and hence do not clump or "stick together" thus remaining diffuse, fluffy, and“halo-like". Hence, galaxies cannot form directly from dark matter.

Moreover, plasma constitutes the major form of matter in the universe [19], most of it invisible. Dark matter being a plasma form of matter seems closer to reality than exotic particles, like WIMPS, MACHOS, etc. [20] whose existence have not been demonstrated.

\section{CONCLUSION}

A quantum model of space and a thermodynamic approach, appear to be useful in understanding our dark universe. In our theory, dark energy is the energy of space and the component of the universe that causes its accelerating expansion. It is associated with a space force which emanate from a scalar field, dubbed as Quintessence. Dark matter, on the other hand, is a plasma form of matter, similar to the state of the universe at the photon epoch, before recombination. It is the precursor in the formation of ordinary matter. Dark energy and dark matter are neither "dark" nor "mysterious", they are just invisible; one is transparent, while the other is opaque. Further work is necessary to better understand the nature and properties of the 
space force, and its associated quantum field. Likewise, we need to understand plasma in space and dark matter halos, along with development of tools to carry them out. Our model brings into fore some questions on long standing concepts in the physical sciences. Space is always associated with energy, thus the concept of vacuum appears outdated; the term "vacuum energy" should be more appropriately called "space energy". Finally, the thermodynamics of expansion of our universe points to its possible origin and ultimate fate. The accelerated expansion of our universe maybe related to a non adiabatic process due to an influx of energy from the "outside". It will be destined to die a cold death if the accelerated expansion continues. Our universe maybe a "bubble" arising from a "mother" universe [21] or the beginning of a new cycle following the "big crunch" of a cyclic universe [22].

\section{REFERENCES}

1. Futch, M., Leibniz, Metaphysics of Time and Space, Springer, NY (2008)

2. Jaffe, R.L, The Casimir Effect and the Quantum Vacuum. in: Physical Review D. 72, (2005)

3. Atkins, P. and De Paula, J. Physical Chemistry, W.H.Freeman, NY (2014)

4. Silk, J. The Big Bang, W.H. Freeman, N.Y. (1989)

5. Weinberg,S., The First Three Minutes, Basic Books, NY (1977)

6. Delsemme, A., Our Cosmic Origins, Cambridge University Press,UK (1998)

7. Kirkwood, J.G. and Oppenheim, I. Chemical Thermodynamics, McGraw-Hill, NY. (1961)

8. Bennett, C.L.; Larson, L.; Weiland, J.L.; Jarosk, N.; et al. "Nine-Year Wilkinson Microwave Anisotropy Probe (WMAP) Observations: Final Maps and Results". The Astrophysical Journal Supplement. 208 (2): 20 (2013)

9. Frieman, J. A.; Turner, M. S.; Huterer, D. "Dark Energy and the Accelerating Expansion of the Universe". Annual Review of Astronomy and Astrophysics. 46 (1): 385-432 (2008)

10. Stenger, V.J., The Comprehensible Cosmos, Prometheus Books, N.Y. (2006)

11. Weinberg, S., Cosmology, Oxford University Press, Oxford, UK (2014)

12. Zee, A., Quantum Field Theory in a Nutshell, $2^{\text {nd }}$ edition, Princeton Univ Press, NJ (2010).

13. Busca, N.G.; Delubac, T; Rich, J., et al, "Baryon Acoustic Oscillations in the Ly- $\alpha$ Forest of BOSS Quasars", Astronomy and Astrophysics, 552, A96 (2013)

14. Siegel, E., personal communication, August 2016

15. Zlatev, I.; Wang, L.; Steinhardt, P. "Quintessence, Cosmic Coincidence, and the Cosmological Constant". Physical Review Letters. 82 (5): 896-899 (1999)

16. Trimble, V. "Existence and nature of Dark matter in the universe". Annual Review of Astronomy and Astrophysics. Palo Alto, CA, Annual Reviews, Inc. 45: 425-472 (1987).

17 De Rujula, A, Jetzer, P, Masso, E, "On the Nature of the Dark Halo of Our Galaxy", Astronomy and Astrophysics, 254, 99, (1992)

18 Dietrich, J., et al, "A filament of dark matter between two clusters of galaxies", Nature 487, 202-204 (2012).

19. Gurnett, D.A.; Introduction to Plasma Physics: With Space and Laboratory Applications, Bhattacharjee, A., Cambridge University Press, UK (2005)

20. Griest, Kim. "The Search for the Dark Matter: WIMPs and MACHOs". Annals of the New York Academy of Sciences. 688, 390-407 (1997)

21 Davis, P., God and the New Physics, Penguin Books, London, UK (1990)

22. Steinhardt, P. J.; Turok, N., A Cyclic Model of the Universe, Science. 296,5572 (2002 